\title{
NOTATIONS ET TERMINOLOGIE
}

- $\mathbb{R}$ est l'ensemble des nombres réels.

- $\mathbb{R}_{+}$est l'ensemble des nombres réels positifs.

- $\overline{\mathbb{R}}$ est la droite réelle achevée, autrement dit, $\overline{\mathbb{R}}=\mathbb{R} \cup\{-\infty,+\infty\}$.

- $\mathbb{Q}$ est l'ensemble des nombres rationnels.

- $\mathbb{Z}$ est l'ensemble des entiers relatifs.

- $\mathbb{N}$ est l'ensemble des entiers naturels.

- $\mathbb{N}^{*}=\mathbb{N} \backslash\{0\}$.

- $[a, b]$ est l'intervalle fermé d'extrémités $a$ et $b$.

- $] a, b[$ est l'intervalle ouvert d'extrémités $a$ et $b$.

- $[x]$ est la partie entière du nombre réel $x$ (on a conservé la notation anglophone).

- Pour $x \in \mathbb{R}$,

$$
\operatorname{sgn} x= \begin{cases}1 & \text { pour } x>0 \\ -1 & \text { pour } x<0 \\ 0 & \text { pour } x=0\end{cases}
$$

- Pour $n \in \mathbb{N}^{*}$,

$$
\begin{aligned}
& n !=1 \times 2 \times 3 \times \cdots \times n, \text { on pose aussi } 0 !=1, \\
& (2 n) ! !=2 \times 4 \times 6 \times \cdots \times(2 n-2) \times 2 n, \\
& (2 n-1) ! !=1 \times 3 \times 5 \times \cdots \times(2 n-3) \times(2 n-1) .
\end{aligned}
$$


- Si $\mathbf{A} \subset \mathbb{R}$ est non vide et majoré, sup $\mathbf{A}$ est alors le plus petit majorant de $\mathbf{A}$. Si l'ensemble non vide $\mathbf{A}$ n'est pas majoré, on pose alors $\sup \mathbf{A}=+\infty$.

- Si $\mathbf{A} \subset \mathbb{R}$ est non vide et minoré, inf $\mathbf{A}$ est alors le plus grand minorant de $\mathbf{A}$. Si l'ensemble non vide $\mathbf{A}$ n'est pas minoré, on pose alors inf $\mathbf{A}=-\infty$.

- Une suite $\left\{a_{n}\right\}$ est dite croissante (resp. décroissante) si $a_{n+1} \geqslant a_{n}$ pour tout $n \in \mathbb{N}$ (resp. $a_{n+1} \leqslant a_{n}$ pour tout $n \in \mathbb{N}$ ). La classe des suites monotones est formée des suites croissantes et des suites décroissantes.

- Soit $\left\{a_{n}\right\}$ et $\left\{b_{n}\right\}$ deux suites réelles $\left(b_{n} \neq 0\right.$ pour tout $\left.n\right)$. Si le quotient $a_{n} / b_{n}$ tend vers 0 (resp. reste borné) lorsque $n$ tend vers $+\infty$, on écrit alors $a_{n}=o\left(b_{n}\right)\left(\right.$ resp. $\left.a_{n}=O\left(b_{n}\right)\right)$.

- Un réel $c$ est une valeur d'adhérence de la suite $\left\{a_{n}\right\}$ s'il existe une sous-suite $\left\{a_{n_{k}}\right\}$ de $\left\{a_{n}\right\}$ qui converge vers $c$.

- Soit $\mathbf{S}$ l'ensemble de toutes les valeurs d'adhérence de $\left\{a_{n}\right\}$. La limite inférieure, $\underset{n \rightarrow+\infty}{\lim } a_{n}$, et la limite supérieure, $\varlimsup_{n \rightarrow+\infty} a_{n}$, sont définies comme suit :

$$
\begin{aligned}
& \varlimsup_{n \rightarrow+\infty} a_{n}= \begin{cases}+\infty & \text { si }\left\{a_{n}\right\} \text { n'est pas majorée, } \\
-\infty & \text { si }\left\{a_{n}\right\} \text { est majorée et } \mathbf{S}=\varnothing, \\
\sup \mathbf{S} & \text { si }\left\{a_{n}\right\} \text { est majorée et } \mathbf{S} \neq \varnothing,\end{cases} \\
& \underline{\lim }_{n \rightarrow+\infty} a_{n}= \begin{cases}-\infty & \text { si }\left\{a_{n}\right\} \text { n'est pas minorée, } \\
+\infty & \text { si }\left\{a_{n}\right\} \text { est minorée et } \mathbf{S}=\varnothing, \\
\inf \mathbf{S} & \text { si }\left\{a_{n}\right\} \text { est minorée et } \mathbf{S} \neq \varnothing .\end{cases}
\end{aligned}
$$

- Un produit infini $\prod_{n=0}^{+\infty} a_{n}$ est dit convergent s'il existe un entier $n_{0} \in \mathbb{N}$ tel que $a_{n} \neq 0$ pour $n \geqslant n_{0}$ et la suite $\left\{a_{n_{0}} a_{n_{0}+1} \cdots \cdots a_{n_{0}+n}\right\}$ converge, lorsque $n$ tend vers $+\infty$, vers une limite $P_{0}$ non nulle. Le nombre $P=a_{1} a_{2} \cdots a_{n_{0}-1} \cdot P_{0}$ est appelée la valeur du produit infini.

- Si $\mathbf{A} \subset \mathbf{X}$ et si $f$ est une fonction définie sur $\mathbf{X}, f_{\mid \mathbf{A}}$ est la restriction de $f$ à $\mathbf{A}$.

$$
\chi_{\mathbf{A}}(x)= \begin{cases}1 & \text { si } x \in \mathbf{A}, \\ 0 & \text { si } x \in \mathbf{X} \backslash \mathbf{A}\end{cases}
$$

est la fonction caractéristique de $\mathbf{A}$. 
- On note $f_{n} \underset{\mathbf{A}}{\rightarrow} f$ pour $\left\{f_{n}\right\}$ converge uniformément vers $f$ sur $\mathbf{A}$.

Si $(\mathbf{X}, d)$ est un espace métrique, $x \in \mathbf{X}$ et $\mathbf{A}$ un sous-ensemble non vide de $\mathbf{X}$, alors

- $\mathbf{A}^{c}=\mathbf{X} \backslash \mathbf{A}$ est le complémentaire de $\mathbf{A}$ dans $\mathbf{X}$,

- $\mathbf{B}_{\mathbf{X}}(x, r), \overline{\mathbf{B}}_{\mathbf{X}}(x, r)$ représentent respectivement la boule ouverte et la boule fermée de centre $x$ et de rayon $r$; si $\mathbf{X}$ est fixé, on omet l'indice et on écrit simplement $\mathbf{B}(x, r), \overline{\mathbf{B}}(x, r)$,

- $\stackrel{\circ}{\mathbf{A}}$ est l'intérieur de $\mathbf{A}$ dans l'espace métrique $(\mathbf{X}, d)$,

- $\overline{\mathbf{A}}$ est l'adhérence de $\mathbf{A}$ dans l'espace métrique,

- $\partial \mathbf{A}=\overline{\mathbf{A}} \cap \overline{\mathbf{X} \backslash \mathbf{A}}$ est la frontière de $\mathbf{A}$,

- $\operatorname{diam}(\mathbf{A})=\sup \{d(x, y): x, y \in \mathbf{A}\}$ est le diamètre de l'ensemble $\mathbf{A}$,

- $\operatorname{dist}(x, \mathbf{A})=\inf \{d(x, y): y \in \mathbf{A}\}$ est la distance de $x$ à l'ensemble $\mathbf{A}$,

- A est un ensemble de type $\mathcal{F}_{\sigma}$ si c'est une union dénombrable d'ensembles fermés dans $(\mathbf{X}, d)$,

- A est un ensemble de type $\mathcal{G}_{\delta}$ si c'est une intersection dénombrable d'ensembles ouverts dans $(\mathbf{X}, d)$,

- $\mathbf{X}$ est connexe s'il n'existe pas de sous-ensembles ouverts disjoints $\mathbf{B}$ et $\mathbf{C}$ de $\mathbf{X}$ tels que $\mathbf{X}=\mathbf{B} \cup \mathbf{C}$.

Continuité, dérivabilité.

- $\mathscr{C}_{\mathbf{A}}$ est l'ensemble des fonctions continues sur A.

- $\mathscr{C}_{] a, b[}^{n}$ est l'ensemble des fonctions $n$ fois continûment dérivables sur $] a, b[$.

- $\mathscr{C}_{[a, b]}^{1}$ est l'ensemble des fonctions continûment dérivables sur $[a, b]$, en considérant aux extrémités respectivement la dérivée à droite et la dérivée à gauche. L'ensemble $\mathscr{C}_{[a, b]}^{n}$ des fonctions $n$ fois continûment dérivables sur $[a, b]$ est défini récursivement.

- $\mathscr{C}_{] a, b[}^{\infty}\left(\right.$ resp. $\left.\mathscr{C}_{[a, b]}^{\infty}\right)$ est l'ensemble des fonctions infiniment dérivables sur $] a, b[$ (resp. $[a, b])$.

Si $f$ et $g$ sont des fonctions réelles d'une variable réelle, alors 
- $f\left(a^{+}\right)$et $f\left(a^{-}\right)$représentent respectivement la limite à droite et la limite à gauche de $f$ en $a$,

- si le quotient $f(x) / g(x)$ tend vers 0 (resp. reste borné) lorsque $x$ tend vers $x_{0}$, on écrit alors $f(x)=o(g(x))$ (resp. $f(x)=O(g(x))$ ),

- $f^{(n)}$ est la dérivée $n$-ième de $f$,

- $f_{+}^{\prime}(a)$ et $f_{-}^{\prime}(a)$ représentent respectivement la dérivée à droite et la dérivée à gauche de $f$ en $a$. 